(C) [2008] IEEE. Reprinted, with permission, from Wang, Zhengguang., Jin, Jianxun., Guo, Youguang., Zhan, Yuedong., \& Zhu, Jianguo. 2008, 'Modelling and Simulation of PMSM Control System Based on SVPWM', Proceedings of the 27th Chinese Control Conference, pp. 724-728. This material is posted here with permission of the IEEE. Such permission of the IEEE does not in any way imply IEEE endorsement of any of the University of Technology, Sydney's products or services. Internal or personal use of this material is permitted. However, permission to reprint/republish this material for advertising or promotional purposes or for creating new collective works for resale or redistribution must be obtained from the IEEE by writing to pubspermissions@ieee.org. By choosing to view this document, you agree to all provisions of the copyright laws protecting it. 


\title{
基于SVPWM的永磁同步电机控制系统模型仿真
}

\author{
王争光 ${ }^{1}$, 金建勋 ${ }^{1}$, 郭有光 ${ }^{2}$, 詹跃东 ${ }^{3,2}$, 朱建国 ${ }^{2}$ \\ 1. 电子科技大学自动化工程学院, 应用超导与电工技术研究中心, 成都 中国 610054 \\ E-mail: zhengguangw@163. com, jxjin@uestc. edu.cn
}

2. 悉尼理工大学工程系, 悉尼, 澳大利亚 2007

E-mail: youguang@eng.uts. edu.au, joe@eng.uts. edu.au.au

\author{
3. 昆明理工大学, 昆明 中国 150001 \\ E-mail: yuedong@eng. uts. edu. au
}

\begin{abstract}
摘 要: 本文简单介绍了电压空间矢量脉宽调制 (SVPWM) 技术的原理, 详细地阐述了在MATLAB/SIMULINK环境下实 现电压空间矢量的方法, 结合永磁同步电机构建了交流伺服控制系统的仿真模型, 仿真波形达到了预期效果, 证明 了该模型的有效性, 同时也为永磁同步电机的软硬件设计提供了理论基础。
\end{abstract}

关键词: 电压空间矢量脉宽调制,永磁同步电机, 建模与仿真

\section{Modeling and Simulation of PMSM Control System Based on SVPWM}

\author{
WANG Zheng-guang ${ }^{1}$, JIN Jian-xun ${ }^{1}$, GUO You-guang ${ }^{2}$, ZHAN Yue-dong ${ }^{3,2}$, and ZHU Jian-guo ${ }^{2}$ \\ 1. Center of Applied Superconductivity and Electrical Engineering, \\ University of Electronic Science and Technology of China, Chengdu, China 610054 \\ E-mail: zhengguangw@163.com jxjin@uestc.edu.cn \\ 2. Faculty of Engineering, University of Technology, Sydney, NSW 2007, Australia \\ E-mail: youguang@eng.uts.edu.au joe@eng.uts.edu.au.au \\ 3. Kunming University of Science and Technology, Kunming, China 150001 \\ E-mail: yuedong@eng.uts.edu.au
}

\begin{abstract}
The paper introduces the basic principle of space vector pulse width modulation simply and expatiates a method for implementing space vector pulse width modulation in detail based on MATLAB/SIMULINK, designs modeling and simulation of AC servo system with PMSM (Permanent Magnet Synchronous Motor), the simulation results show that the model is effective, and the method provides a base for both software and hardware design of an actual PMSM.
\end{abstract}

Key words: Space vector pulse width modulation, PMSM, modeling and simulation

\section{1 引言(Introduction)}

随着电力电子技术、微型计算机技术、稀土永磁 材料和控制理论的飞速发展, 交流电机的数字化控制 越来越被广泛地应用。空间矢量脉宽调制（Space Vector Pulse Width Modulation, SVPWM) 是根据变频 器空间电压矢量切换来控制变频器。电压空间矢量 PWM控制策略由日本学者在20世纪80年代针对交流电 机变频调速提出的, 其主要思路是采用逆变器空间矢 量电压的切换以获得准圆形旋转磁场, 从而使逆变器 输出适当波形的电压, 这就是磁链轨迹跟踪脉宽调制 的基本原理 ${ }^{[1-6]}$ 。电压空间矢量脉宽调制的出发点与

此项工作属国家863资助项目, 项目批准号:2007AA03Z208
正弦脉宽矢量调制（Sine Pulse Width Modulation, SPWM）不同, SPWM调制是从三相交流电压出发, 其着眼点是如何生成一个可以调压调频的三相对称 正弦电压波形。而SVPWM是将逆变器和电动机看成 一个整体，用八个基本电压矢量合成期望的电压矢 量, 建立逆变器功率器件的开关状态, 并依据电机磁 链和电压的关系, 从而实现对电动机恒磁通变压变频 调速。若忽略定子电阻压降, 当定子绕组施加理想的 正弦电压时, 由于电压空间矢量为等幅的旋转矢量, 故气隙磁通以恒定的角速度旋转, 轨迹为圆形 ${ }^{[7-8]}$ 。 本文针对具有体积小、重量轻、效率高、转动惯量小、 可靠性高等优点的永磁同步电机所建立的PMSM交 流伺服控制系统的数学模型, 能更好实现永磁同步电 机交流伺服系统数字化控制。 


\section{SVPWM原理 (Principle of SVPWM)}

SVPWM是通过逆变器功率器件的不同开关模 式产生有效电压矢量来逼近基准圆，图1是一个典型 的三相电压源逆变器模型 ${ }^{[9]}$ 。对于三相电压型逆变器 电路, 每相桥臂都可以被看作一个开关 $\mathrm{S}$, 开关函数 $\mathrm{S}_{\mathrm{A}}(\mathrm{t}) 、 \mathrm{~S}_{\mathrm{B}}(\mathrm{t}) 、 \mathrm{~S}_{\mathrm{C}}(\mathrm{t})$ 表示三个上桥臂的开关状态。

相应的逆变器输出电压空间矢量可表示为

$$
\mathrm{V}\left(\mathrm{S}_{\mathrm{A}}, \mathrm{S}_{\mathrm{B}}, \mathrm{S}_{\mathrm{C}}\right)=2 \mathrm{~V}_{\mathrm{dc}}\left(\mathrm{S}_{\mathrm{A}}+\alpha \mathrm{S}_{\mathrm{B}}+\alpha^{2} \mathrm{~S}_{\mathrm{C}}\right) / 3
$$

$\mathrm{V}_{\mathrm{dc}}$ 其中为变频器的直流母线上的电压, $\alpha=\mathrm{e}^{\mathrm{j} 120}$ 。如 果把上桥臂功率开关器件的导通状态用 “ 1 ” 表示, 关断用 “0” 表示, 上桥臂三个功率开关器件的开关 状态共有八种组合，构成了对应的八个电压空间矢 量, 如下图2所示 ${ }^{[10-11]}$ 。图中 $V_{0}(000)$ 和 $V_{7}(111)$ 称为零 电压空间矢量, 其余六个矢量称为有效矢量, 其模为 $2 V_{d c} / 3$, 若 $V$ 在 $I$ 区时, 则 $V$ 可以由 $V_{4} 、 V_{6} 、 V_{0}$ 和 $V_{7}$ 合成, 依据平行四边形法则, 有

$$
\mathrm{T}_{4} \mathrm{~V}_{4} / \mathrm{T}+\mathrm{T}_{6} \mathrm{~V}_{6} / \mathrm{T}=\mathrm{TV}_{\text {out }}
$$

逆变器的八种开关状态如表1所示。

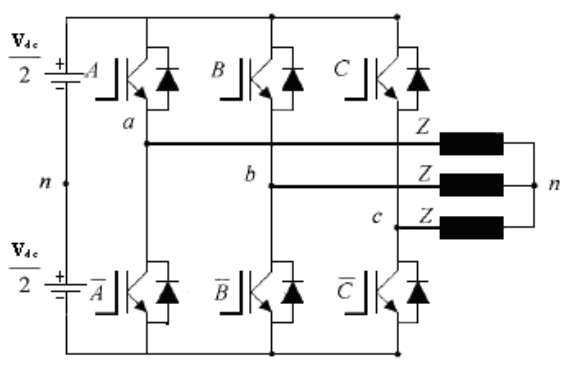

图1：逆变器模型

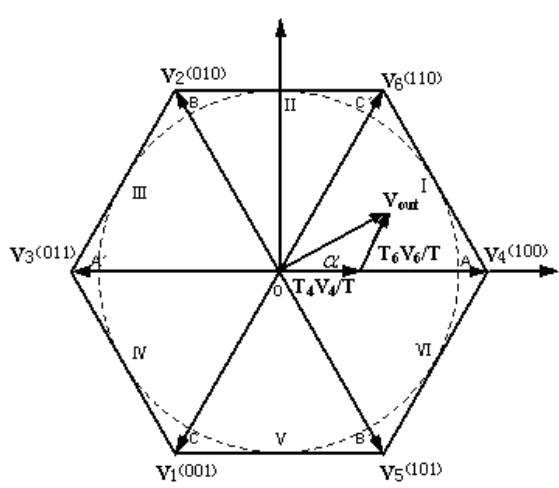

图2：两电平电压空间矢量

表1: 逆变器的八种开关状态

\begin{tabular}{c|c|c|c|c}
\hline \hline 逆变器状态 & $\mathrm{S}_{\mathrm{A}} \mathrm{S}_{\mathrm{B}} \mathrm{S}_{\mathrm{C}}$ & $\mathrm{V}_{\mathrm{A}} / \mathrm{V}_{\mathrm{dc}}$ & $\mathrm{V}_{\mathrm{B}} / \mathrm{V}_{\mathrm{dc}}$ & $\mathrm{V}_{\mathrm{C}} / \mathrm{V}_{\mathrm{dc}}$ \\
\hline 0 & 000 & 0 & 0 & 0 \\
\hline 1 & 001 & $-1 / 3$ & $-1 / 3$ & $2 / 3$ \\
\hline 2 & 010 & $-1 / 3$ & $2 / 3$ & $-1 / 3$ \\
\hline 3 & 011 & $-2 / 3$ & $1 / 3$ & $1 / 3$ \\
\hline 4 & 100 & $2 / 3$ & $-1 / 3$ & $-1 / 3$ \\
\hline 5 & 101 & $1 / 3$ & $-2 / 3$ & $1 / 3$ \\
\hline 6 & 110 & $1 / 3$ & $1 / 3$ & $-2 / 3$ \\
\hline 7 & 111 & 0 & 0 & 0 \\
\hline \hline
\end{tabular}

\section{SVPWM的SIMULINK仿真 (Simulation of SVPWM with SIMULINK)}

由SVPWM的原理可知, 产生SVPWM波形的仿 真模块主要包括: 扇区判断模块、计算基本矢量的作 用时间模块、开关作用时间的计算模块、SVPWM波 形的生成模块等。

\section{1 扇区判断(Estimation of Sector)}

在应用SVPWM技术时, 首先要确定合成电压矢 量所处的扇区, 由于矢量的直角坐标形式适合矢量控 制, 所以扇区的确定采用如下方法。

扇区与 $\mathrm{V}_{\alpha}, \mathrm{V}_{\beta}$ 的关系有: 当 $\mathrm{V}_{\beta}>0$ 时, 令 $\mathrm{A}=1$; 当 $\sqrt{3} \mathrm{~V} \alpha-\mathrm{V} \beta>0$ 时, 令 $\mathrm{B}=1$; 当 $\sqrt{3} \mathrm{~V} \alpha+\mathrm{V} \beta<0$ 时, 令 $\mathrm{C}=1$ 。由 $\mathrm{N}=\mathrm{A}+2 \mathrm{~B}+4 \mathrm{C}$ 可知, 扇区与 $\mathrm{N}$ 的对应关系如表 1 , 模型如图3所示。

表 2：扇区与 $\mathrm{N}$ 的对应关系

\begin{tabular}{c|c|c|c|c|c|c}
\hline \hline 扇区 & I & II & III & IV & V & VI \\
\hline $\mathrm{N}$ & 3 & 1 & 5 & 4 & 6 & 2 \\
\hline \hline
\end{tabular}

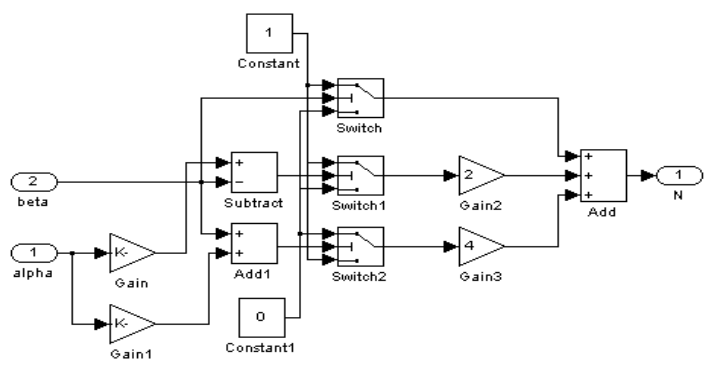

图3：扇区判断模型

\section{2 计算基本矢量的作用时间(Calculation of Effect Time of Basic Vector)}

令 $\mathrm{Z}=\mathrm{T}\left(-\sqrt{3} \mathrm{~V}_{\alpha}+\mathrm{V}_{\beta}\right) /\left(\sqrt{2} \mathrm{~V}_{\mathrm{dc}}\right), \mathrm{Y}=\mathrm{T}\left(\sqrt{3} \mathrm{~V}_{\alpha}+\right.$ $\left.\mathrm{V}_{\beta}\right) /\left(\sqrt{2} \mathrm{~V}_{\mathrm{dc}}\right), \mathrm{X}=2 \mathrm{~T} \mathrm{~V} \beta /\left(\sqrt{2} \mathrm{~V}_{\mathrm{dc}}\right)$, 则 $\mathrm{N}$ 与矢量作 用时间 $\mathrm{T}_{1}$ 和 $\mathrm{T}_{\mathrm{m}}$ 的对应关系如表3所示, 计算模型如图 4所示。

表3：基本矢量作用时间

\begin{tabular}{c|c|c|c|c|c|c}
\hline \hline $\mathrm{N}$ & 1 & 2 & 3 & 4 & 5 & 6 \\
\hline $\mathrm{T}_{1}$ & $\mathrm{Z}$ & $\mathrm{Y}$ & $-\mathrm{Z}$ & $-\mathrm{X}$ & $\mathrm{X}$ & $-\mathrm{Y}$ \\
\hline $\mathrm{T}_{\mathrm{m}}$ & $\mathrm{Y}$ & $-\mathrm{X}$ & $\mathrm{X}$ & $\mathrm{Z}$ & $-\mathrm{Y}$ & $-\mathrm{Z}$ \\
\hline \hline
\end{tabular}

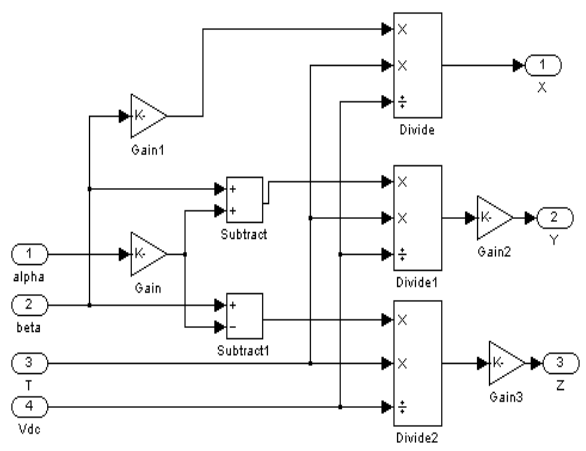

图4：计算X, Y, Z模型 
由于 $\mathrm{T}_{1}$ 和 $\mathrm{T}_{\mathrm{m}}$ 之和一定要小于或等于 $\mathrm{T}(\mathrm{PWM}$ 调制 周期), 所以还要进行过饱和判断, 当 $\mathrm{T}_{1}+\mathrm{T}_{\mathrm{m}}>\mathrm{T}$ 时, 应取: $\mathrm{T}_{1}=\mathrm{T}_{1} \times \mathrm{T} /\left(\mathrm{T}_{1}+\mathrm{T}_{\mathrm{m}}\right), \mathrm{T}_{\mathrm{m}}=\mathrm{T}_{\mathrm{m}} \times \mathrm{T} /\left(\mathrm{T}_{1}+\mathrm{T}_{\mathrm{m}}\right)$, 其 SIMULINK实现模型如图5所示。

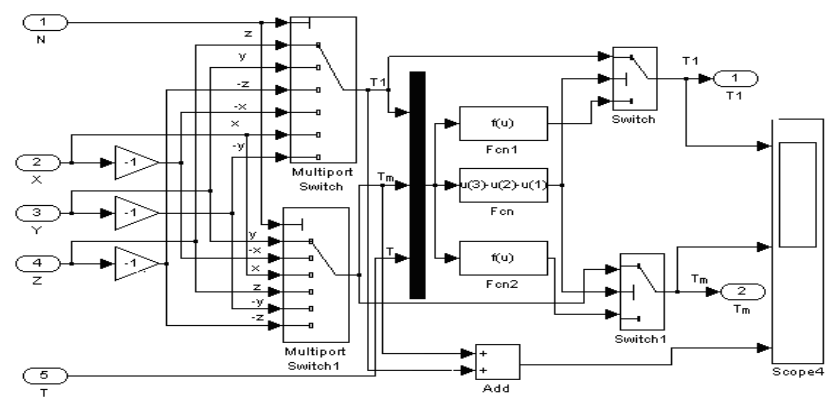

图5：基本矢量作用时间计算模型

\section{3 开关作用时间的计算模型(Calculational Model of Switch Effect Time)}

令 $\mathrm{T}_{\mathrm{a}}=\left(\mathrm{T}-\mathrm{T}_{1}-\mathrm{T}_{\mathrm{m}}\right) / 4, \mathrm{~T}_{\mathrm{b}}=\mathrm{T}_{\mathrm{a}}+\mathrm{T}_{1} / 2, \mathrm{~T}_{\mathrm{c}}=\mathrm{T}_{\mathrm{b}}+\mathrm{T}_{\mathrm{m}} / 2$, 其 模型如图6所示, 则 $\mathrm{N}$ 与开关作用时间 $\mathrm{T}_{\mathrm{cm} 1} 、 \mathrm{~T}_{\mathrm{cm} 2} 、 \mathrm{~T}_{\mathrm{cm} 3}$ 之间的对应关系如表4所示。

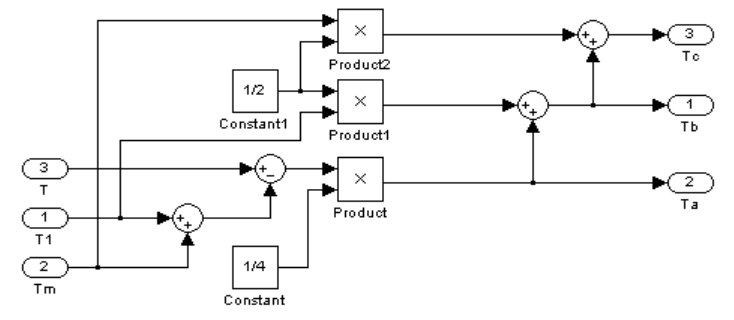

图6：开关作用时间
表 4: $\mathrm{T}_{\mathrm{cm}}$ 与 $\mathrm{T}_{\mathrm{a}} 、 \mathrm{~T}_{\mathrm{b}} 、 \mathrm{~T}_{\mathrm{c}}$ 对应关系表

\begin{tabular}{c|c|c|c|c|c|c}
\hline \hline $\mathrm{N}$ & 1 & 2 & 3 & 4 & 5 & 6 \\
\hline $\mathrm{T}_{\mathrm{cm} 1}$ & $\mathrm{~T}_{\mathrm{b}}$ & $\mathrm{T}_{\mathrm{a}}$ & $\mathrm{T}_{\mathrm{a}}$ & $\mathrm{T}_{\mathrm{c}}$ & $\mathrm{T}_{\mathrm{c}}$ & $\mathrm{T}_{\mathrm{b}}$ \\
\hline $\mathrm{T}_{\mathrm{cm} 2}$ & $\mathrm{~T}_{\mathrm{a}}$ & $\mathrm{T}_{\mathrm{c}}$ & $\mathrm{T}_{\mathrm{b}}$ & $\mathrm{T}_{\mathrm{b}}$ & $\mathrm{T}_{\mathrm{a}}$ & $\mathrm{T}_{\mathrm{c}}$ \\
\hline $\mathrm{T}_{\mathrm{cm} 3}$ & $\mathrm{~T}_{\mathrm{c}}$ & $\mathrm{T}_{\mathrm{b}}$ & $\mathrm{T}_{\mathrm{c}}$ & $\mathrm{T}_{\mathrm{a}}$ & $\mathrm{T}_{\mathrm{b}}$ & $\mathrm{T}_{\mathrm{a}}$ \\
\hline \hline
\end{tabular}

\subsection{SVPWM波形的生成(Waveform Generation of SVPWM)}

计算得到的 $\mathrm{T}_{\mathrm{cm} 1} 、 \mathrm{~T}_{\mathrm{cm} 2}$ 和 $\mathrm{T}_{\mathrm{cm} 3}$ 值与等腰三角形进 行比较, 就可以生成对称空间矢量PWM波形 ${ }^{[12-14]}$, 模 型如图7所示。

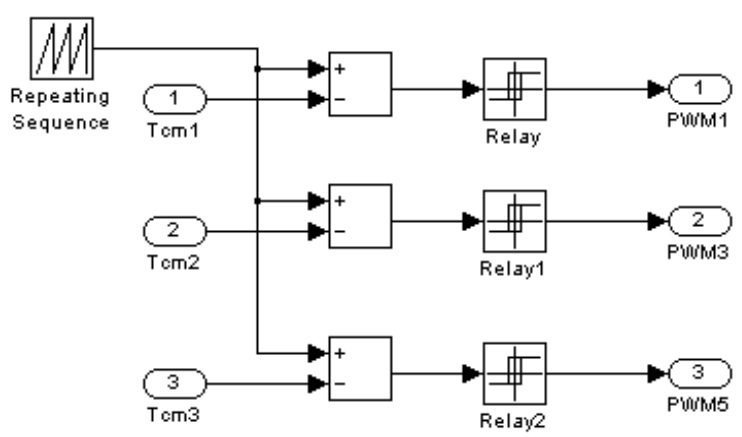

图7：PWM波型的生成模型

将生成的PWM1、PWM3和PWM5进行非运算就 可以生成PWM2、PWM4和PWM6，从而控制开关管 的通断来控制永磁同步电机, 将上述模块连接生成如 下图8所示的SVPWM整体模型。

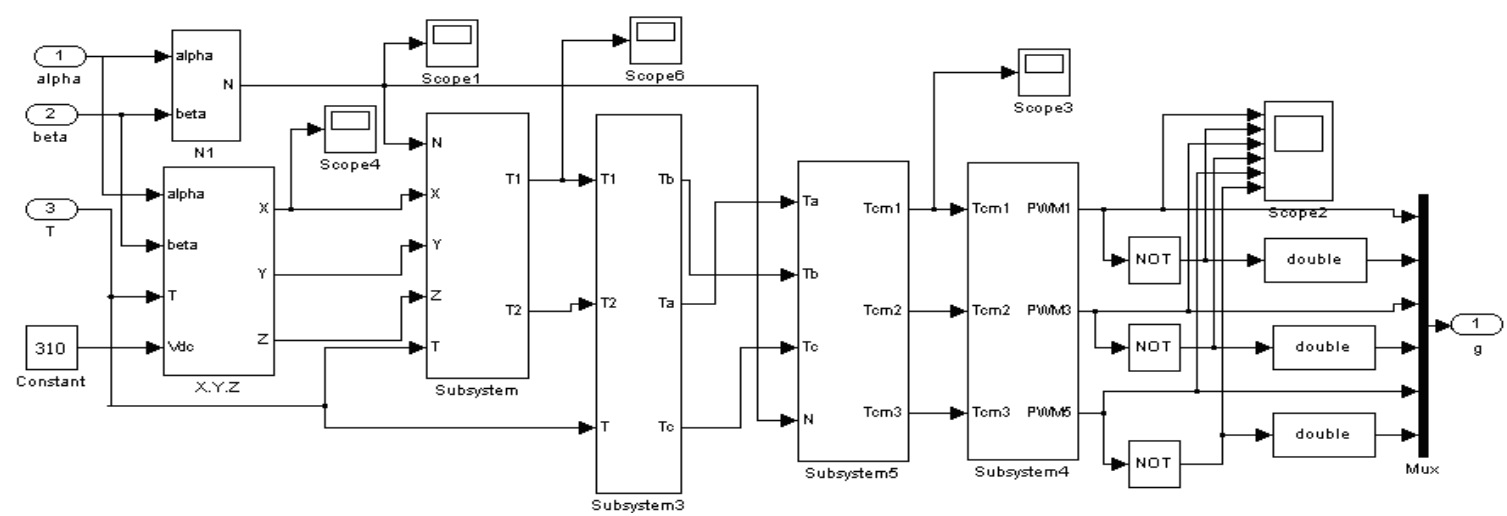

图8：SVPWM模型

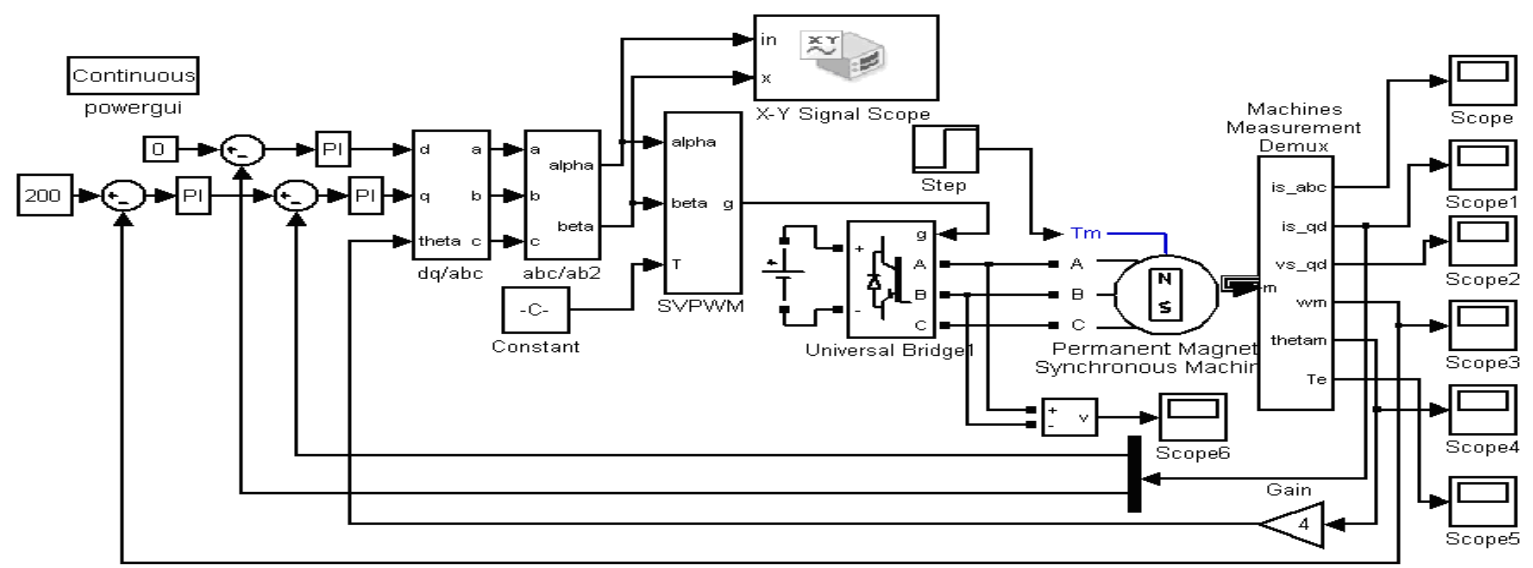

图9：PMSM磁场定向控制系统仿真模型 


\section{4 永磁同步电机仿真模型的建立 (Modeling and Simulation of PMSM )}

将上述各个子模块进行连接构成闭环系统的仿 真模型。由于测量模块输出的转角为机械角度, 转 速为机械角速度, 而实际坐标变换中采用的是电角 度, 所以要把所测量的角度值乘以电机的极对数得 到电角度。所建控制系统的仿真模型见图9。

\section{5 仿真结果 (Simulation Results)}

给定PWM采样周期为 $0.0001 \mathrm{~s}$, 直流母线电压为 $310 \mathrm{~V}$ 时, $\mathrm{PWM}$ 载波频率为 $10 \mathrm{kHz}$, 死区时间为 $4.1 \mu \mathrm{s}$, 一个采样周期内的SVPWM波形如图10所示, 其合成 转矩增加了 $\sqrt{3}$ 倍, 每隔 $60^{\circ}$ 电角度换相一次, 每个
功率管 $120^{\circ}$ 电角度, 每个绕组通电 $240^{\circ}$ 电角度, 其 中正向和反向通电各 $120^{\circ}$ 电角度。

电机的电流、转速、转矩、转角也符合电机的 实际运行特性, 证明了所建模型的正确性。速度调 节器参数设置: $\mathrm{K}_{\mathrm{P}}=1.5, \mathrm{~K}_{\mathrm{i}}=10.5 ; \mathrm{q}$ 轴电流调节器 参数设置: $\mathrm{K}_{\mathrm{p}}=3, \mathrm{~K}_{\mathrm{i}}=1 ; \mathrm{d}$ 轴电流调节器参数设置 为 $\mathrm{K}_{\mathrm{p}}=3, \mathrm{~K}_{\mathrm{i}}=1$, 在调整好参数的永磁同步电机仿真 系统电流、转速、转矩、转角如图11所示。仿真给 定速度为 $200 \mathrm{rad} / \mathrm{s}$, 在 $\mathrm{t}=0 \mathrm{~s}$ 时刻, 电机空载起动, 在 $\mathrm{t}=0.2 \mathrm{~s}$ 时突加 $3 \mathrm{Nm}$ 的负载转矩, 仿真时间为 $0.4 \mathrm{~s}$, 仿 真步长为 $0.0001 \mathrm{~s}$ 。由此可见, 电机启动速度很快, 能快速跟踪给定速度, 在加载情况下, 转速波动很 小。本例仿真中用到的为TYB系列的永磁同步电机, 型号为TYB55-4, 其参数如表5所示。

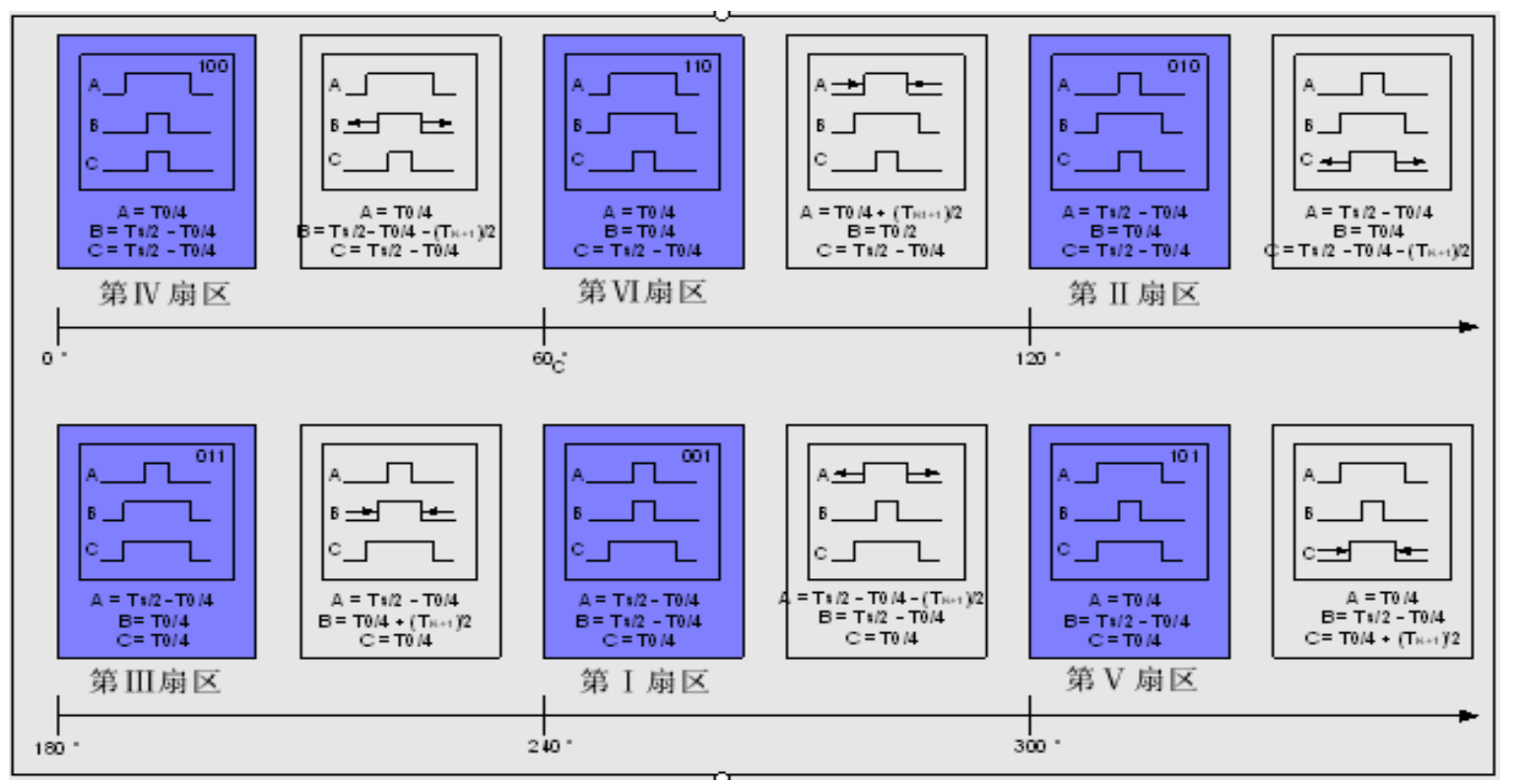

图10：单个采样周期内的SVPWM波形

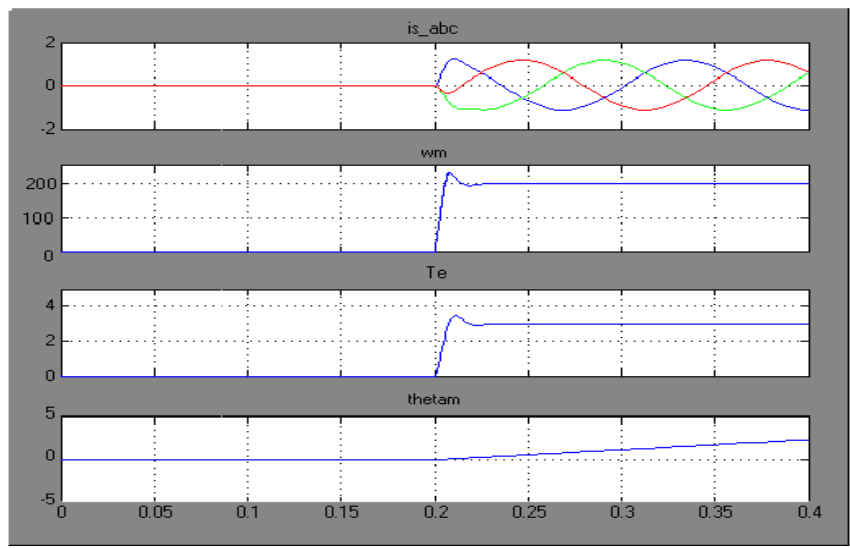

图11: 电流、转速、转矩、转角

图12为 $\mathrm{d}-\mathrm{q}$ 轴定子电流仿真波形图, 当 $\mathrm{t}=0.2 \mathrm{~s}$ 时, 转矩从 0 变为 $3 \mathrm{Nm}, \mathrm{q}$ 轴电流与转矩成正比, $\mathrm{d}$ 轴电流 接近于零, 由此可见三相定子电流实现了很好的解 耦。
表5：永磁同步电机参数

\begin{tabular}{c|c}
\hline \hline 电机功率 & $200 \mathrm{~W}$ \\
\hline 转动惯量 & $0.8 \mathrm{kgm}^{2}$ \\
\hline 额定转矩 & $3.5 \mathrm{Nm}$ \\
\hline 额定电压 & $380 \mathrm{~V}$ \\
\hline 额定电流 & $1.5 \mathrm{~A}$ \\
\hline 定子电阻 & $2.875 \Omega$ \\
\hline 额定感抗 & $8.5 \mathrm{mH}$ \\
\hline 额定转速 & $1500 \mathrm{rpm}$ \\
\hline 调频范围 & $10-50 \mathrm{~Hz}$ \\
\hline \hline
\end{tabular}

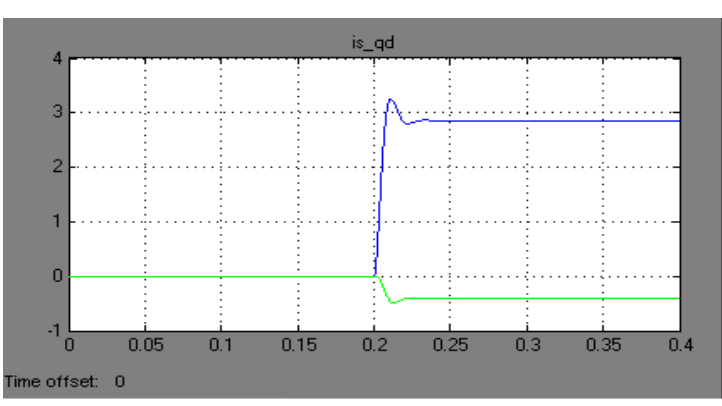

图12：d-q轴定子电流仿真波形图 


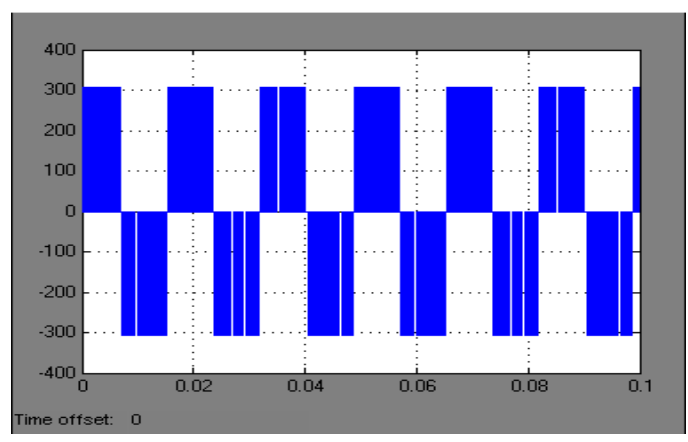

图13：线电压仿真波形

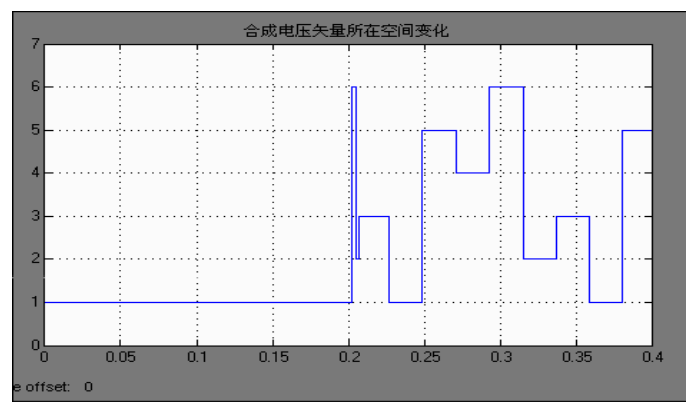

图14：合成电压矢量所在扇区变换

在仿真时给定速度 $200 \mathrm{rad} / \mathrm{s}$, 图13为电机 $\mathrm{AB}$ 线 电压的仿真波形, 图14为合成电压矢量所在扇区变 换, 可见电压矢量依次转过III (011)， I (001), V (101), IV (100), VI (110), II (010), 电压矢量逆时针旋转, 如图14所示图形与预期结果 图10完全一致。从转速响应曲线可以看出, 转速在 起动之后, 很快达到稳定值。在转矩响应曲线中, 在起动时刻, 电磁转矩很快稳定在设定值 $3 \mathrm{Nm}$, 并 有轻微波动, ia、ib、ic相电流曲线和转矩响应曲线 有些相似, 在开始时刻, 电流值比较大, 但很快达 到设定值。

\section{6 结论 (Conclusion)}

本文在分析永磁同步电机的转子磁场定向控制 的基础上, 在Matlab/Simulink环境下, 采用矢量控 制与经典的速度、电流双闭环控制方法建立了永磁 同步电机控制系统的仿真模型, 仿真实验结果表明: 波形符合理论分析，系统能平稳运行，并具有较好 的静、动态特性, 尤其为新发展的高温超导磁悬浮 飞轮储能和高温超导直线电机装置的控制系统的软 硬件设计奠定了理论基础 ${ }^{[15-17]}$ 。SVPWM比SPWM的 电压利用率高15\%, 这是两者最大的区别, SVPWM 是一种在SPWM的相调制波中加入了零序分量后进 行规则采样得到的结果, SPWM易于硬件电路实现, 而SVPWM更适合于数字化控制系统。

\section{参考文献}

[1] 李崇坚, 交流同步电机调速系统, 科学出版社, 北京, 2007.
[2] Y. K. Chin, J. Soulard, A Permanent Magnet Synchronous Motor for Traction Applications of Electric Vehicles, IEEE International Electric Machines and Drives Conference, 1035-1041, 2003.

[3] D. G. Xu, H. Wang, J. Z. Shi, PMSM Servo System with Speed and Torque Observer, Annual Power Electronics Specialists Conference, Vol.34, No.1, 241-245, 2003.

[4] Q. H. Liu, M. A. Jabbar, A. M. Khambadkone, Design Optimization of Wide-speed Permanent Magnet Synchronous Motors, International Conference on Power Electronics, Machines and Drives, University of Bath, UK, 404-408, 2002.

[5] Y. L. Xu, J. Q. Xu, W. B. Wan, R. Y .Tang, Development of Permanent Magnet Synchronous Motor Used in Electric Vehicle, 5th International Conference on Electrical Machines and Systems (ICEMS'2001), Shenyang, China, Vol.2, 884-887, August 18-20, 2001.

[6] J. Q. Xu, Y. L. Xu, R. Y .Tang, Development of Full Digital Control System for Permanent Magnet Synchronous Motor Used in Electric Vehicle, 5th International Conference on Electrical Machines and Systems (ICEMS'2001), Shenyang, China, Vol.1, 554-556, August 18-20, 2001.

[7] B. T. Ooi, J. C. Salmon, J. W. Dixon, A. B. Kulkarni, A Three Phase Controlled-current PWM Converter with Leading Power Factor, IEEE Trans. Ind. Application, Vol. IA-23, No.1, 78-84, Jan/Feb, 1987.

[8] J. W. Dixon, B. T. Ooi, Indirect Current Control of a Unity Power Factor Sinusoidal Current Boost Type Three-phase Rectifier, IEEE Trans. Ind. Electron., Vol.35, No.4, 508-515, Nov, 1988.

[9] B. Vladimir, K. Vikram, A New Mathematical Model and Control of a Three-Phase AC-DC Voltage Source Converter, IEEE Trans. on Power Electronics, Vol.12, No.1, 116-123, January, 1997.

[10] R. Wu, S. B. Dewan, G. R. Slemon, A PWM AC to DC Converter with Fixed Switching Frequency, in Conf. Rec. I988 IEEE-IAS Ann. Mag., 706-711.

[11] R. Wu, S. B. Dewan, G. R. Slernon, Analysis of an AC-to-DC Voltage Source Converter Using PWM with Phase and Amplitude Control, IEEE Trans. on Industry Applications, Vol.27, No.2, March/April, 1991.

[12] M. Kadjoudj, M .E. H. Benbouzid, A Robust Hybrid Current Control for Permanent Magnet Synchronous Motor Drive, IECON'01, 2068-2073, 2001.

[13] J. Dixon, S. Tepper, L. Moran, Practical Evaluation of Different Modulation Technique for Current-controlled Voltage Source Inverters, IEEE Proceedings Electric Power Applications, Vol.143, No.4, 301-306, 1996.

[14] B. D. Fernando, D. W. Michael, L. D. Robert, Dynamic Analysis of Current Regulators for AC Motors Using Complex Vectors, IEEE Trans. on Industry Application, Vol.35, No.6, 1424-1432 , 1999.

[15] J. X. Jin, Y. G. Guo, J. G. Zhu, Principle and Analysis of a Linear Motor Driving System for HTS Levitation Applications, Physica C, Vol.460-462, 1445-1446, 2007.

[16] Y. G. Guo, J. X. Jin, J. G. Jian, H. Y. Lu, Design and Analysis of a Prototype Linear Motor Driving System for HTS Maglev Transportation, IEEE Transaction on Applied Superconductivity, Vol.17, No.2, 2087-2090, June, 2007.

[17] J. X. Jin, Y. G. Guo, J. X. Chen, L. H. Zheng, J. G. Zhu, HTS Levitation and Transportation with Linear Motor Control, Proceedings of the 26th Chinese Control Conference, Zhangjiajie, Hunan, China, Vol.6, 10-14, July, 2007. 